\title{
Implementation of Protective Protocols during COVID-19 Pandemic and Older Patients' Outcomes from the Department of Oral and Maxillofacial Surgery, Chulalongkorn University, Thailand
}

\author{
Palinee Hongpaitoon $^{1,2}$, Nithimar Sermsuti-anuwat ${ }^{3}$ \& Kiti Siriwatana ${ }^{4}$ \\ ${ }^{1}$ Master of Science Program in Oral and Maxillofacial Surgery, Department of Oral and Maxillofacial Surgery, \\ Faculty of Dentistry, Chulalongkorn University, Bangkok, Thailand \\ ${ }^{2}$ Chatturat Hospital, Nong Bua Yai, Chatturat District, Chaiyaphum Province, Thailand \\ ${ }^{3}$ Academic Affairs Division, Faculty of Dentistry, Chulalongkorn University, Bangkok, Thailand \\ ${ }^{4}$ Department of Oral and Maxillofacial Surgery, Faculty of Dentistry, Chulalongkorn University, Bangkok, \\ Thailand \\ Correspondence: Kiti Siriwatana, Department of Oral and Maxillofacial Surgery, Faculty of Dentistry, \\ Chulalongkorn University, 34 Henri-Dunant Road, Wangmai, Pathumwan, Bangkok 10330, Thailand. Tel: \\ +66-218-8648. E-mail: kiti.s@chula.ac.th
}

Received: August 12, 2021 Accepted: November 4, 2021 Online Published: November 5, 2021

doi:10.5539/gjhs.v13n12p51

URL: https://doi.org/10.5539/gjhs.v13n12p51

\begin{abstract}
Objectives: According to COVID-19 pandemic in Thailand, the Faculty of Dentistry, Chulalongkorn University (DCU) implemented the protective protocols across all groups of people, with no specific protocols for the elderly patents. This study aimed to review those protocols and its impact on the older patients' treatment outcomes in the OMFS clinic, DCU and identify the potential areas for improvement.
\end{abstract}

Methods: This observational study based on secondary data was conducted using the DCU-COVID-19 protocols and outcome data obtained from the DCU online database from March 26 to May 31, 2020. The descriptive approach was used for data analysis.

Results: According to the DCU guidelines, all clinics strictly adhered to standard precautions, all health personnel were required to use personal protective equipment (PPE). Of the 853 older patients who visited the DCU, 35 (4.1\%) received urgency or emergency treatments at the OMFS clinic; 19 (54.3\%) were females, and $16(45.7 \%)$ were males (mean age, 71.03 years). The most common chief complaint was pain $(\mathrm{n}=23 ; 65.7 \%)$. No reports of COVID-19 transmission were received among dental staff and patients in the DCU. Additionally, none of the elderly patients returned to the clinic complaining of recurrence or worsening of symptoms or without a scheduled appointment.

Conclusions: The DCU-COVID-19 protective protocols for all patients, including older patients who attended the OMFS, appear to be appropriate and effective. However, telephone-based recall after surgical treatment at the clinic should be used, particularly for older patients.

Keywords: COVID-19, oral and maxillofacial surgery, personal protective equipment, triage, geriatrics

\section{Introduction}

The new infectious disease (2019-nCoV or COVID-19) caused by a novel coronavirus was originally reported in Wuhan City, China, in December 2019 and rapidly spread to other countries (WHO, 2020). The outbreak is currently a major challenging public health issue across the world (Wang, Horby, Hayden, \& Gao, 2020).

The Center for Disease Control and Prevention (CDC) and World Health Organization (WHO) updated the information on the modes of COVID-19 transmission by early 2021; the disease is known to be spread by inhaling aerosol particles; depositing respiratory droplets and particles on exposed mucous membranes in the eye, nose, or mouth via sprays and splashes; and touching mucous membranes with hands either directly or indirectly by touching surfaces with the virus on them (CDC, 2020; WHO, 2020; Greenhalgh, Jimenez, Prather, Tufekci, Fisman, \& Schooley, 2021). Both symptomatic and asymptomatic patients with COVID-19 can carry and act as the 
main sources of transmission (Chan, Yuan, Kok, To, Chu, Yang et al., 2020; Johansson, Quandelacy, Kada, Prasad, Steele, Brooks et al., 2021).

Most dental procedures in the clinic can lead to the generation of splatters and aerosols contaminated with microorganisms, saliva, and blood. Therefore, the risk of spreading infection is higher among dental practitioners and patients when compared to other non-aerosol-generating procedures (Harrel \& Molinari, 2004; Mehta, Pulluri \& Karibasappa, 2015; Ampornaramveth, 2017). Aerosol-generating procedures are associated with the highest risk of COVID-19 transmission. The aerosols and droplets are produced when dental instruments, such as rotary instruments, ultrasonic scalers, air polishers, and air abrasion units, are used. The particles are mixed with the infected patient's saliva or blood and can act as sources of infection. Aerosols, in particular, consist of small particles (diameter, $<5 \mu \mathrm{m}$ ) that can stay airborne for a longer time. Furthermore, the reduced distance between the dental personnel and the patient increases the risk of disease transmission (Peng, $\mathrm{Xu}, \mathrm{Li}$, Cheng, Zhou, \& Ren, 2020; Meng, Hua, \& Bian, 2020).

Consequently, dental management has to be altered to accommodate the current pandemic situation. Appropriate management within the dental setting can reduce the risk of infections, including COVID-19. The development of a clinical management guideline for dental procedures during the ongoing pandemic is of utmost importance (ADA, 2020).

The virus is continuing to spread across the world, and Thailand is going through the next wave of the pandemic. According to the Ministry of Public Health of Thailand, the number of confirmed COVID-19 cases and deaths until July 31 , 2021, was 597,287 and 4,857, respectively; more than $70 \%$ of the deaths were among patients aged $\geq 60$ years. Approximately $20 \%$ of the elderly population in Thailand has been vaccinated (WHO Thailand, 2020). Globally, several deaths associated with COVID-19 have been recorded among older patients with underlying systemic health conditions. Most of the individuals in the population group are frail and suffer from chronic systemic diseases (Yanez, Weiss, Romand, \& Treggiari, 2020).

However, the oral health of older people in Thailand is generally worse than that of the younger population, and most of the elderly present with dental caries and periodontal disease (MOPH, 2017). Poor oral health among the elderly can negatively impact their quality of life, general health, and diet, thus exacerbating the underlying medical conditions. A recent study evaluated the impacts of restrictions to the provision of dental services for this population group during the COVID-19 pandemic and reported a significant reduction in oral healthcare provision for dependent older adults (Lundberg, Hillebrecht, Mckenna, \& Srinivasan, 2021).

The Thailand government enforced the first emergency decree on March 26, 2020 (GOVT, 2020). The Department of Medical Services of the Ministry of Public Health and Dental Council developed certain dental practice guidelines for the COVID-19 pandemic (DMS, 2020a; DMS, 2020b; DAT, 2020; DDC, 2020).

According to the mentioned evidence and Thailand's policies on the current pandemic, the Faculty of Dentistry at Chulalongkorn University (DCU) has been requested to suspend all routine dental treatments during the present situation (DCU, 2020). The available DCU protective protocols are implemented in all clinics across all groups of people, with no specific protocols for the elderly.

This study aims to review our experiences in managing older patients in the oral and maxillofacial surgery (OMFS) clinic at the DCU under the available protective protocols during the COVID-19 pandemic. Additionally, the impacts of these protocols on the outcomes in this population and the areas for further improvement were evaluated.

\section{Method}

\subsection{Study Design and Setting}

This observational study based on secondary data was conducted at the OMFS clinic located in the DCU, Thailand. The DCU-COVID-19 protective protocols and outcome data from the faculty's online database during the first wave of the COVID-19 pandemic were used in the study. This study protocol was approved by the human research ethics committee of the DCU on October 2, 2020 (study code: HREC-DCU 2020-088, approval no. 097/2020). No informed consent was needed as the data collection involved a secondary database and all patient identifiers, if any collected, were removed.

\subsection{Study Population and Sample Size}

A total of 835 patients (age, $\geq 60$ years), who received dental treatment at the DCU during the COVID-19 pandemic during the first emergency decree (from March 26 to May 31, 2020), were enrolled in this study. Older patients with complete data were included in the study, whereas those with missing data were excluded. 


\subsection{Data Collection and Data Analysis}

The secondary data were collected into two parts. The first part involved the gathering of announcements, such as protective protocols, policies, and guidelines, at the DCU during the first wave of the COVID-19 pandemic. The second part comprised the secondary data of the outcomes of the patients, including demographic and general information, dental problem or chief complaint, treatment received, recall visit, and other details, recorded in the DCU online database from March 26 to May 31, 2020. The descriptive approach was used for data analysis.

\section{Results}

\section{The announced protocols of the dental services in the DCU during the COVID-19 pandemic}

The DCU has been regulating the dental services (DCU, 2020) according to the National Guidelines for Dental Practice to reduce the effect of the pandemic by providing treatment for patients who present with dental emergencies or conditions that required urgent dental care, in concordance with the American Dental Association (ADA). According to the ADA, dental emergency refers to "a potentially life-threatening condition that requires immediate treatment, such as uncontrolled bleeding, cellulitis, a diffuse soft-tissue bacterial infection with intraoral or extraoral swelling or trauma involving the facial bones that can potentially compromise the airway." The term "urgent dental care" refers to conditions requiring immediate attention and should be treated using minimally invasive techniques, e.g., severe dental pain from pulpal inflammation, pericoronitis or third-molar pain, surgical postoperative osteitis or dry socket dressing changes, abscess or localized bacterial infection resulting in localized pain and swelling, tooth fracture resulting in pain or causing soft-tissue trauma, dental trauma with avulsion/luxation, and dental treatment required prior to critical medical procedures (such as radiotherapy, chemotherapy, and transplantation) (ADA, 2020).

The DCU recognized the unprecedented and extraordinary circumstances faced by dentists and all healthcare professionals regarding their growing concerns about COVID-19. The provision of all routine and nonurgent dental treatments has been restricted, and only dental emergencies can be treated according to the DCU recommendations. All clinics are required to strictly adhere to standard precautions, which remain in the same way as the normal practice. The guidelines include using personal protective equipment (PPE), surface cleaning, and other precautions in accordance with the principles of transmission-based precautions. The guidelines provided by the faculty at the DCU are summarized in Tables 1 and 2 (DCU, 2020).

Table 1. Summary of the standard and transmission-based precautions translated from the DCU guidelines

\begin{tabular}{|c|c|c|}
\hline Infection control & & \\
\hline \multirow{8}{*}{ Standard precautions } & & 1. Hand hygiene \\
\hline & & 2. PPE use \\
\hline & & 3. Reprocessing of reusable instruments and equipment \\
\hline & & 4. Surface cleaning \\
\hline & & 5. Appropriate handling of linen \\
\hline & & 6. Safety regarding sharp objects \\
\hline & & 7. Waste management \\
\hline & & 8. Respiratory hygiene and cough etiquette \\
\hline \multirow{7}{*}{ Transmission-based precautions } & \multirow{2}{*}{ Contact } & 1. Maintain a distance of at least 3 feet \\
\hline & & 2. Healthcare personnel must wear a gown and gloves \\
\hline & \multirow{2}{*}{ Droplet } & 1. Maintain a distance of at least 3 feet \\
\hline & & 2. Healthcare personnel must wear a mask \\
\hline & \multirow{3}{*}{ Airborne } & 1. Administrative management \\
\hline & & 2. Environmental management \\
\hline & & 3. Respiratory protection for healthcare personnel \\
\hline
\end{tabular}


Table 2. Necessary precautions are categorized as "need" to indicate those that need to be followed and "optional," which include additional guidelines that enhance safety, if followed

\begin{tabular}{|c|c|c|c|c|}
\hline Precaution & & Important measure & Need & Optional \\
\hline \multirow{2}{*}{ Standard precautions } & & PPE & $\sqrt{ }$ & \\
\hline & & Surface cleaning & $\sqrt{ }$ & \\
\hline \multirow{8}{*}{$\begin{array}{l}\text { Droplet and airborne } \\
\text { precautions }\end{array}$} & \multirow{3}{*}{ Administrative management } & $\begin{array}{l}\text { Preappointment risk assessment } \\
\text { (online, telephone) }\end{array}$ & & $\sqrt{ }$ \\
\hline & & $\begin{array}{l}\text { Patient screening at appointment } \\
\text { date }\end{array}$ & $\sqrt{ }$ & \\
\hline & & COVID-19 rapid test & & $\sqrt{ }$ \\
\hline & \multirow{4}{*}{ Environmental management } & Negative pressure room & $\begin{array}{r}\sqrt{ } \\
\text { (high } \\
\text { risk) }\end{array}$ & $\sqrt{ }$ \\
\hline & & Ventilation system 6-12 ACH & $\sqrt{ }$ & \\
\hline & & $\begin{array}{l}\text { High-efficiency particulate air } \\
\text { (HEPA) filtration }\end{array}$ & $\sqrt{ }$ & \\
\hline & & Ultraviolet germicidal irradiation & & $\sqrt{ }$ \\
\hline & $\begin{array}{l}\text { Respiratory protection for } \\
\text { healthcare personnel }\end{array}$ & N95 or fit face mask & $\sqrt{ }$ & \\
\hline \multirow{5}{*}{ Additional precautions } & & Rubber dam & $\sqrt{ }$ & \\
\hline & & $\begin{array}{l}\text { Hand-free aerosol suction system, } \\
\text { e.g., EasyPrep }{ }^{\circledR}\end{array}$ & & $\sqrt{ }$ \\
\hline & & High-power evacuation & $\sqrt{ }$ & \\
\hline & & Extraoral air suction & & $\sqrt{ }$ \\
\hline & & Preoperative antiseptic mouthwash & $\sqrt{ }$ & \\
\hline
\end{tabular}

\section{The management of patients in oral and maxillofacial surgery during COVID-19 pandemic}

The management in the DCU is divided into three main parts: (1) patient management before treatment, (2) patient management during the treatment process, and (3) postoperative management (DCU, 2020).

\section{(1) Patient management before treatment at the DCU}

\section{Appointment}

Dental students or staff assess the patient's risk via telephone, then confirms the appointment and emphasizes the submission of the results of the risk assessment on the day scheduled for the appointment. If the result indicates a high risk, the appointment is postponed. The DCU used social media to give information, assess the level of emergency, and suggest self-care recommendations for each patient. In this manner, the number of patients who do not need emergency treatment are screened, and the total number of patients who visit the clinic is reduced.

\section{Patient screening and triaging}

The OMFS clinic provides treatment at the Somdet Ya Building at the DCU during the pandemic situation. The body temperature is determined first using a contact-free forehead thermometer before the patient enters the building. A questionnaire is used to assess the risk for COVID-19 (Table 3). 
Table 3. The questionnaire used for screening

Due to the current COVID-19 situation, we request you to provide us with the most accurate and truthful medical statements so that we can provide you with the appropriate treatment and help in preventing the spread of the disease effectively.

Please circle your answer.

\begin{tabular}{ll} 
1. Have you or a person close to you traveled or from a foreign country within the past 14 days? & Yes or No \\
$\begin{array}{l}\text { 2. Have you or anyone close to you visited a place or community affected by the COVID-19 outbreak } \\
\text { within the past } 14 \text { days? }\end{array}$ & Yes or No \\
$\begin{array}{l}\text { 3. Have you or anyone close to you developed any symptoms such as fever, runny nose, cough, sore } \\
\text { throat, shortness of breath, or loss of taste or smell within the past 14 days? }\end{array}$ & Yes or No \\
\hline $\begin{array}{l}\text { 4. Have you been close to a patient with suspected or confirmed COVID-19 within the past 14 days? } \\
\text { 5. Have you recovered from COVID-19 within the past 30 days? }\end{array}$ & Yes or No \\
\hline I certify that the above information is correct and true in every aspect.
\end{tabular}

\section{(2) Patient management during the treatment processes}

\section{Personal protective equipment (PPE)}

The types of appropriate PPE to be used by dental clinic workers have been classified based on the occupational exposure risk level (Table 4).

Table 4. Appropriate PPE for dentists classified according to the risk of occupational exposure to SARS-CoV-2

\begin{tabular}{|c|c|}
\hline Services & PPE \\
\hline Office workers & Surgical mask \\
\hline Staff at screening checkpoints or examination rooms & Surgical mask, face shield \\
\hline Dentists' assistants who work in support of the clinic & Gown, surgical mask, face shield \\
\hline $\begin{array}{l}\text { Dentists and assistants who provide examination or } \\
\text { procedures not involving aerosol-generating procedures }\end{array}$ & Gown, surgical mask, face shield, hair net, disposable gloves \\
\hline $\begin{array}{l}\text { Dentists and assistants who provide aerosol-generating } \\
\text { procedures }\end{array}$ & $\begin{array}{l}\text { Gown, waterproof gown/isolation gown, face shield, hair net, } \\
\text { disposable gloves, boot cover/shoe cover, surgical mask } \\
\text { (peripheral seal with tape or with mask fitter) or N95, face shield }\end{array}$ \\
\hline $\begin{array}{l}\text { Dentists and assistants who treat patients with suspected } \\
\text { or confirmed COVID-19 }\end{array}$ & $\begin{array}{l}\text { Scrubs, gown, disposable isolation gown/medical protective } \\
\text { coverall, medical cap/hood, boot cover/shoe cover, N95, } \\
\text { goggles/face shield }\end{array}$ \\
\hline
\end{tabular}

\section{Preprocedural antiseptic mouth rinsing}

The DCU's guidelines recommend the use of an antimicrobial mouthwash, such as $0.2 \%$ chlorhexidine rinse, by the patient before the dental procedure. Moreover, all staff should follow the guidelines to increase their safety during this situation as mentioned in Table 5. 
Table 5. Guidelines for increasing safety during dental practice as summarized from the DCU guidelines

\begin{tabular}{|c|c|}
\hline Procedure & Practice guideline for safety \\
\hline \multirow{3}{*}{ Oral examination and non-aerosol-generating procedure } & $\begin{array}{l}\text { 1. Ensure that all patients use a preprocedural mouth rinse for } \\
20-30 \text { s before the treatment. }\end{array}$ \\
\hline & $\begin{array}{l}\text { 2. Avoid using instruments such as a triple syringe, which } \\
\text { generate aerosols. }\end{array}$ \\
\hline & 3. Avoid triggering a cough or sneeze. \\
\hline \multirow{6}{*}{ Aerosol-generating procedure } & $\begin{array}{l}\text { 1. Ensure that all patients use a preprocedural mouth rinse for } \\
20-30 \text { s before the treatment. }\end{array}$ \\
\hline & 2. Avoid triggering a cough or sneeze. \\
\hline & 3. Use rubber dam isolation, if possible. \\
\hline & $\begin{array}{l}\text { 4. Consider using a hand-free aerosol suction system, such as } \\
\text { EasyPrep®. }\end{array}$ \\
\hline & 5. Use high-volume evacuation effectively. \\
\hline & 6. Use the four-hand technique to reduce contamination. \\
\hline \multirow{7}{*}{$\begin{array}{l}\text { Very high-risk procedures (patients with respiratory disease } \\
\text { symptoms and need dental emergency care) }\end{array}$} & $\begin{array}{l}\text { 1. Assess dental emergencies and provide treatment for } \\
\text { emergency cases only (specialty clinic } 3 \text { ). }\end{array}$ \\
\hline & $\begin{array}{l}\text { 2. Ensure that all patients use a preprocedural mouth rinse for } 1 \\
\text { min before the treatment. }\end{array}$ \\
\hline & 3. Avoid triggering a cough or sneeze. \\
\hline & 4. Use rubber dam isolation, if possible. \\
\hline & $\begin{array}{l}\text { 5. Consider using a hand-free aerosol suction system, such as } \\
\text { EasyPrep }{ }^{\circledR} \text {, in cases where the rubber dam cannot be placed. }\end{array}$ \\
\hline & 6. Use high-volume evacuation effectively. \\
\hline & 7. Use the four-hand technique to reduce contamination. \\
\hline
\end{tabular}

\section{(3) Postoperative management}

Instrument processing: Standard precautions should be used to collect and deliver the instruments for sterilization at the DCU's Central Sterile Supply Department.

Surface cleaning: Contaminated surfaces should be thoroughly cleaned with a disinfectant wipe using two or more wipes; areas that are not very dirty should also be wiped.

Air management: Sufficient airflow should be provided within the treatment area for at least 10 min before the next patient is treated. Open the window for ventilation during lunch break and after completion of work for at least 10 15 min every day. While providing treatment to patients with high risk at the specialty clinic, the window should be left open for ventilation during the patient's alteration, or collect the equipment after treatment for at least 15 min.

\section{The Elderly Patients' Treatment Outcomes}

\section{General characteristics and medical records of patients}

Table 6 presents the demographic characteristics and clinical features of the study participants. A total of 35 older patients (mean age, $71.03 \pm 7.402$ years) who received emergency or urgent treatment in the OMFS clinic were enrolled in this study; $19(54.3 \%)$ of them were females, and $16(45.7 \%)$ were males. The patients were divided into two age groups as follows: those $\leq 68$ years $(n=19 ; 54.3 \%)$ and those above 68 years of age $(n=16 ; 45.7 \%)$. More than half of the patients reported the presence of a systemic disease ( $\mathrm{n}=22 ; 62.9 \%)$.

During the period of this study, the most chief complaint was pain $(n=23 ; 65.7 \%)$, and the vast majority $(n=34$; $97.1 \%$ ) of the patients received urgent dental treatment. The most common type of procedure used was non-aerosol-generating $(\mathrm{n}=33 ; 94.3 \%)$. Furthermore, 32 patients $(91.4 \%)$ had no future appointments scheduled for recall. Only three patients had a recall appointment, and all three of them presented with normal wound healing, improvement in their clinical condition, and no worsening of symptoms during the recall visit. Moreover, none of 
the patients returned to the clinic with their previous chief complaint, worsening of symptoms, or without an appointment.

Table 6. Demographic characteristics and clinical features of the study participants $(\mathrm{N}=35)$

\begin{tabular}{|c|c|c|}
\hline Continuous variables & Mean (SD) & Median (Min-Max) \\
\hline Age (years) & $71.03( \pm 7.402)$ & $68(61-84)$ \\
\hline Categorical variables & Number & Percentage \\
\hline \multicolumn{3}{|l|}{ Gender } \\
\hline Female & 19 & 54.3 \\
\hline Male & 16 & 45.7 \\
\hline \multicolumn{3}{|l|}{ Age } \\
\hline$\leq 68$ years & 19 & 54.3 \\
\hline$>68$ years & 16 & 45.7 \\
\hline \multicolumn{3}{|l|}{ Medical condition } \\
\hline Denied any systemic diseases & 13 & 37.1 \\
\hline Has some systemic diseases & 22 & 62.9 \\
\hline \multicolumn{3}{|l|}{ Chief complaints } \\
\hline Pain & 23 & 65.7 \\
\hline Swelling with pain & 5 & 14.3 \\
\hline Cracked/fractured teeth & 3 & 8.6 \\
\hline Others & 4 & 11.4 \\
\hline \multicolumn{3}{|l|}{ Type of treatment } \\
\hline Urgent & 34 & 97.1 \\
\hline Emergency & 1 & 2.9 \\
\hline \multicolumn{3}{|l|}{ Type of procedure } \\
\hline Non aerosol & 33 & 94.3 \\
\hline Aerosol & 2 & 5.7 \\
\hline \multicolumn{3}{|l|}{ Appointment for recall } \\
\hline No & 32 & 91.4 \\
\hline Yes & 3 & 8.6 \\
\hline \multicolumn{3}{|c|}{ Clinical improvement on recall visits } \\
\hline Yes & 3 & 100 \\
\hline No & 0 & 0 \\
\hline \multicolumn{3}{|c|}{ Return without an appointment } \\
\hline No & 35 & 100 \\
\hline Yes & 0 & 0 \\
\hline
\end{tabular}

Abbreviations: SD, Standard Deviation; min-max, minimum-maximum.

\section{Areas for Improvement}

Although the DCU has administered teleconsultation prior to the admission of patients to the OMFS clinic during the pandemic period, it does not provide regular telephone-based recalls for all patients after they have visited the clinic. Consequently, improvements in the outcomes of the treatment could not be guaranteed among the older patients. Therefore, a telephone-based recall system after surgical treatment used for elderly and vulnerable 
patients might prove useful in monitoring their health.

\section{Discussion}

The guidelines set by the DCU during the COVID-19 pandemic emphasized factors such as the selection of cases and the application of measures for infection control. The main protocols follow the national measures outlined in the interim guidelines from the ADA (DMS, 2020a; DMS, 2020b; DAT, 2020; DDC, 2020; ADA, 2020). The body temperatures of all patients, including older patients and their bystanders, are determined before entering the clinic, and the patients are required to undergo the COVID-19 risk assessment before receiving the treatment. Patients and visitors are required to wear a mask when they are in the hospital. Only patients with dental emergencies were treated; nonurgent cases were postponed (DCU, 2020).

Despite the absence of any specific protocols for older patients, none of the elderly patients treated between March 26 and May 31,2020, returned to the clinic with any other complaints or without an appointment scheduled by the dentist. However, improvements in the treatment outcomes could not be guaranteed in these patients. We believe that the DCU should include teledentistry before and after the patient visits the OMFS. Gebczynski et al. concluded that teledentistry reduces the costs and restrictions in obtaining access to dental care and is a promising tool that provides easy access to consultations with specialists, especially among patients from dispersion and excluded groups Gebczynski, Zarzecka, \& Słowik, 2020). Similarly, Rahman et al. recommended telehealth as a method of consultation, which would help in the recovery of elective services and reduce the spread of COVID-19 (Rahman, Nathwani, \& Kandiah, 2020).

In the current study, there were no reports of COVID-19 transmission among the dental staff or patients in the DCU. The number of staff at the OMFS clinic has been reduced; all of them were required to regularly monitor themselves for fever and other symptoms consistent with COVID-19 and report the results of the COVID-19 risk assessment before they provide treatments. Only dental emergency treatments were provided, and aerosol-generating procedures were avoided, as far as possible. The case selection criteria were in accordance with the dental emergency guidelines set by the ADA (DMS, 2020a; DMS, 2020b; DAT, 2020; DDC, 2020; ADA, 2020).

The OMFS clinic strictly applied the standard and additional precautions related to contact, droplet, and airborne transmission based on the type of procedure required and the associated occupational risk. As previously reported (Meng, Hua, \& Bian, 2020; Guo, Cao, Hong, Tan, Chen, Jin et al., 2020). COVID-19 transmission occurs mainly when an individual comes in close contact with another infected person, such as a family member or a healthcare worker. Healthcare workers need to provide the treatment in accordance with the updated protocols for safety purposes. The Occupational Safety and Health Administration (OSHA) suggested that dental staff in the reported COVID-19 cluster areas should wear N95 masks or other effective respirators that can protect them from both non aerosol- and aerosol-generating procedures (OSHA, 2020). A lack of N95 masks, the reuse of PPE, and suboptimal hand hygiene practices were considered as risk factors for COVID-19 infection (Guo, Wang, Hu, Wu, Gu, Wang et al., 2020; Ran, Chen, Wang, Wu, Zhang, \& Tan, 2020).

The OMFS clinic at the DCU maintains social distancing by preparing the waiting area with seats that are placed at least 6 feet apart. Air purifiers with HEPA filters were used for the removal of airborne particles (Liu, Ning, Chen, Guo, Liu, Gali et al., 2020).

\subsection{Limitations and Suggestions}

The main limitation of this study is the use of a secondary database during the first wave of the COVID-19 pandemic in Thailand. However, it was conducted to answer the proposed objectives. Nonetheless, additional studies must be conducted during the peak of any future waves, and results need to be compared with those of the current study to confirm our findings.

\section{Conclusions}

The results of this study showed that the DCU guidelines for the management of all patients, including older patients, who attended the OMFS clinic during the COVID-19 pandemic were appropriate and effective. According to the strict protocols, no reports of COVID-19 transmission were reported among the dental staff and patients at the DCU. Additionally, none of the elderly patients came back to the clinic with any further complaints or without an appointment. However, a telephone-based recall system after treatment might aid in accurately determining the outcomes of the treatment among elderly patients. The experiences and clinical protocols described in this study might prove helpful for other oral and maxillofacial units in a similar academic environment during this pandemic. 


\section{Acknowledgements}

The authors thank to all contribute people that give facilitate for this research.

\section{Funding}

The authors received no financial support for the research, authorship, and/or publication of this article.

\section{Competing Interests Statement}

The Authors declare that there is no conflict of interest.

\section{References}

ADA, American Dental Association. (2020). Summary of ADA guidance during the COVID-19 crisis. Retrieved from

https://www.ada.org/en/press-room/news-releases/2020-archives/april/summary-of-ada-guidance-during-the -covid-19-crisis

Ampornaramveth, R. (2017). Air quality in dental clinic. Journal of the dental association of Thailand, 67(1), $1-14$.

CDC, Centers of Disease Control and Prevention. (2020). Infection control guidance for healthcare professionals about coronavirus (COVID-19). Retrieved from https://www.cdc.gov/coronavirus/2019-nCoV/hcp/infection-control.html

Chan, J. F.-W., Yuan, S., Kok, K.-H., To, K. K.-W., Chu, H., Yang, J., .. Yuen, K.-Y. (2020). A familial cluster of pneumonia associated with the 2019 novel coronavirus indicating person-to-person transmission: a study of a family cluster. The Lancet, 395(10223), 514-523. https://doi.org/10.1016/S0140-6736(20)30154-9

DAT, The Dental Association of Thailand. (2020). Guidelines for infection control and prevention in dental settings in the situation of COVID-19 pandemic. Retrieved from https://www.thaidental.or.th/main/download/upload/upload-204211344051371.pdf

DCU, Faculty of Dentistry, Chulalongkorn University. (2020). Guidelines for dental treatment during the spread of COVID-19 at Faculty of Dentistry, Chulalongkorn University. Retrieved from http://www.dent.chula.ac.th/upload/images2/hospital/13_hospitaldocument.pdf

DDC, Department of Disease Control. (2020). Case definition for patients under investigation (PUIs) with COVID-19 (edited March 2, 2020). Retrieved from https://ddc.moph.go.th/viralpneumonia/eng/file/guidelines/G_PUIdefinition.pdf

DMS, Department of Medical Services, Ministry of Public Health Thailand. (2020a). Guideline of relief measures for dental treatment in the situation of COVID-19 pandemic. Retrieved from http://dentalcouncil.or.th/images/uploads/file/MF5PQXQLIC0PBD17.pdf.

DMS, Department of Medical Services, Ministry of Public Health Thailand. (2020b). Dental treatment in the situation of the outbreak of COVID-19. Retrieved from http://dentalcouncil.or.th/images/uploads/file/5F7VSJ8HPWBYTSNS.pdf

Gebczynski, K. A., Zarzecka, J., \& Słowik, J. (2020). Teledentistry as potential opportunity for the improvement of dental care in periods of social isolation: preliminary results of literature review. Journal of Stomatology, 73(6), 351-354. https://doi.org/10.5114/jos.2020.102054

Government of Thailand, Ministry of Foreign Affairs. (2020) Regulation Issued under Section 9 of the Emergency Decree on Public Administration in Emergency Situations B.E. 2548 (2005) (No. 1). Retrieved from https://www.mfa.go.th/en/content/115867-regulation-issued?page $=5 \mathrm{f} 22514 \mathrm{~b} 78568958 \mathrm{aa} 0 \mathrm{~d} 5 \mathrm{~b} 85 \& \mathrm{menu}=5 \mathrm{~d}$ $5 \mathrm{bd} 3 \mathrm{cb} 15 \mathrm{e} 39 \mathrm{c} 306002 \mathrm{a} 9 \mathrm{~b} 9$

Greenhalgh, T., Jimenez, J. L., Prather, K. A., Tufekci, Z., Fisman, D., \& Schooley, R. (2021). Ten scientific reasons in support of airborne transmission of SARS-CoV-2. Lancet (London, England), 397(10285), 1603-1605. https://doi.org/10.1016/S0140-6736(21)00869-2

Guo, X., Wang, J., Hu, D., Wu, L., Gu, L., Wang, Y., Zhao, J., Zeng, L., Zhang, J., \& Wu, Y. (2020). Survey of COVID-19 Disease Among Orthopaedic Surgeons in Wuhan, People's Republic of China. The Journal of bone and joint surgery, American volume, 102(10), 847-854. https://doi.org/10.2106/JBJS.20.00417

Guo, Y.-R., Cao, Q.-D., Hong, Z.-S., Tan, Y.-Y., Chen, S.-D., Jin, H.-J., ... Yan, Y. (2020). The origin, transmission and clinical therapies on coronavirus disease 2019 (COVID-19) outbreak - an update on the status. Military 
Medical Research, 7(1). https://doi.org/10.1186/s40779-020-00240-0

Harrel, S. K., \& Molinari, J. (2004). Aerosols and splatter in dentistry. The Journal of the American Dental Association, 135(4), 429-437. https://doi.org/10.14219/jada.archive.2004.0207

Johansson, M. A., Quandelacy, T. M., Kada, S., Prasad, P. V., Steele, M., Brooks, J. T., ... Butler, J. C. (2021). SARS-CoV-2 Transmission From People Without COVID-19 Symptoms. JAMA Network Open, 4(1), e2035057. https://doi.org/10.1001/jamanetworkopen.2020.35057

Liu, Y., Ning, Z., Chen, Y., Guo, M., Liu, Y., Gali, N. K., ... Lan, K. (2020). Aerodynamic analysis of SARS-CoV-2 in two Wuhan hospitals. Nature, 582(7813), 557-560. https://doi.org/10.1038/s41586-020-2271-3

Lundberg, A., Hillebrecht, A., Mckenna, G., \& Srinivasan, M. (2021). COVID-19: Impacts on oral healthcare delivery in dependent older adults. Gerodontology, 38(2), 174-178. https://doi.org/10.1111/ger.12509

Mehta, D. S., Pulluri, P., \& Karibasappa, S. N. (2015). Aerosol and splatter in Dentistry - An Overview. CODS Journal of Dentistry, 7(1), 28-32. https://doi.org/10.5005/cods-7-1-28

Meng, L., Hua, F., \& Bian, Z. (2020). Coronavirus Disease 2019 (COVID-19): Emerging and Future Challenges for Dental and Oral Medicine. Journal of Dental Research, 99(5), 481-487. https://doi.org/10.1177/0022034520914246

Meng, L., Hua, F., \& Bian, Z. (2020). Coronavirus Disease 2019 (COVID-19): Emerging and Future Challenges for Dental and Oral Medicine. Journal of Dental Research, 99(5), 481-487. https://doi.org/10.1177/0022034520914246

MOPH, Ministry of Public Health. (2017). National oral health survey of Thailand 2017. Retrieved from http://www.dent.chula.ac.th/upload/news/791/file_1_5834.pdf.

OSHA. Occupational Safety and Health Administration. (2020). Dentistry workers and employers 2020.

Peng, X., Xu, X., Li, Y., Cheng, L., Zhou, X., \& Ren, B. (2020). Transmission routes of 2019-nCoV and controls in dental practice. International Journal of Oral Science, 12(1). https://doi.org/10.1038/s41368-020-0075-9

Rahman, N., Nathwani, S., \& Kandiah, T. (2020). Teledentistry from a patient perspective during the coronavirus pandemic. British Dental Journal. https://doi.org/10.1038/s41415-020-1919-6

Ran, L., Chen, X., Wang, Y., Wu, W., Zhang, L., \& Tan, X. (2020). Risk Factors of Healthcare Workers With Coronavirus Disease 2019: A Retrospective Cohort Study in a Designated Hospital of Wuhan in China. Clinical Infectious Diseases, 71(16), 2218-2221. https://doi.org/10.1093/cid/ciaa287

Wang, C., Horby, P. W., Hayden, F. G., \& Gao, G. F. (2020). A novel coronavirus outbreak of global health concern. The Lancet, 395(10223), 470-473. https://doi.org/10.1016/S0140-6736(20)30185-9

WHO Thailand, World Health Organization. (2020). COVID-19-WHO Thailand situation reports. Retrieved from https://www.who.int/thailand/emergencies/novel-coronavirus-2019/situation-reports.

World Health Organization. [WHO]. (2020). Coronavirus disease (COVID-2019) situation reports. Retrieved from https://www.who.int/emergencies/diseases/novel-coronavirus-2019/situation-reports.

Yanez, N. D., Weiss, N. S., Romand, J.-A., \& Treggiari, M. M. (2020). COVID-19 mortality risk for older men and women. BMC Public Health, 20(1). https://doi.org/10.1186/s12889-020-09826-8

\section{Copyrights}

Copyright for this article is retained by the author(s), with first publication rights granted to the journal.

This is an open-access article distributed under the terms and conditions of the Creative Commons Attribution license (http://creativecommons.org/licenses/by/4.0/). 